\title{
Enhanced diapycnal mixing due to near-inertial internal waves propagating through an Anticyclonic eddy in the ice-free Chukchi Plateau
}

\author{
Kawaguchi ${ }^{1}$, Y., S. Nishino ${ }^{1}$, J. Inoue ${ }^{2}$, K. Maeno ${ }^{3}$, H. Takeda $1^{4}$, and K. Oshima ${ }^{1}$ \\ ${ }^{1}$ Japan Agency for Marine-Earth Science and Technology, Yokosuka, Japan \\ ${ }^{2}$ Natitional Institute of Polar Research, Tokyo, Japan \\ ${ }^{3}$ Nippon Marine Enterprises, Yokosuka, Japan \\ ${ }^{4}$ Tokyo Gakugei Univ., Tokyo, Japan
}

The Arctic Ocean is known to be quiescent in terms of turbulent kinetic energy (TKE) associated with internal waves. To investigate the current state of TKE in the seasonally ice-free Chukchi Plateau, Arctic Ocean, this study performed a 3-week, fixed-point observation (FPO) using repeated microstructure, hydrographic, and current measurements in September 2014. During the FPO program, the microstructure observation detected noticeable peaks of TKE dissipation rate $\varepsilon$ during the transect of an anticyclonic eddy moving across the FPO station. Particularly, $\varepsilon$ had a significant elevation in the lower halocline layer, near the critical level, reaching the order of $10^{-8} \mathrm{~W} \mathrm{~kg}{ }^{-1}$. The ADCP-measured current displayed energetic near-inertial internal waves (NIWs) propagating via the stratification at the top and bottom of the anticyclone. According to spectral analyses of horizontal velocity, the waves had almost downward energy propagation, and its current amplitude reached $\sim 10 \mathrm{~cm} \mathrm{~s}^{-1}$. The WKB scaling, incorporating vertical variations of relative vorticity, suggests that increased wave energy near the two pycnoclines was associated with diminishing group velocity at the corresponding depths. The fine-scale parameterization using observed near-inertial velocity and buoyancy frequency successfully reproduced the characteristics of observed $\varepsilon$, supporting that the near-inertial kinetic energy can be effectively dissipated into turbulence near the critical layer. According to a mixed layer slab model, a rapidly moving storm that has passed over in the first week likely delivered the bulk of NIW kinetic energy, eventually captured by the vortex, into the surface water.
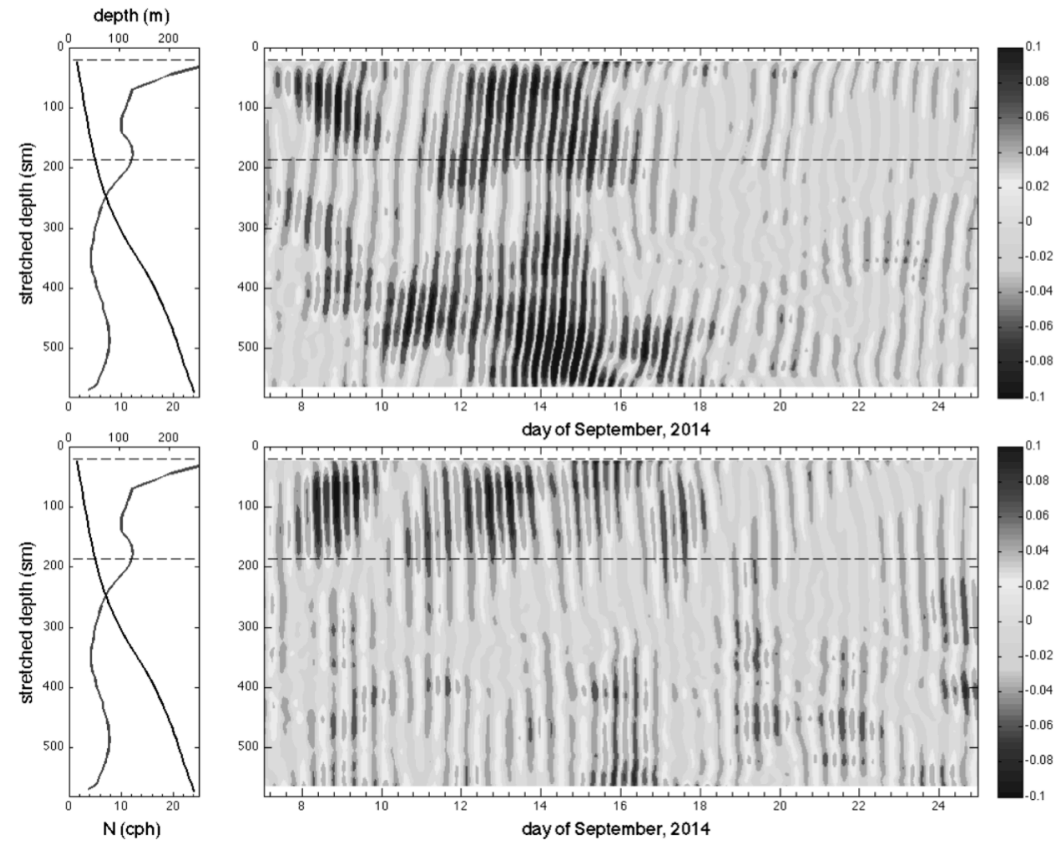

Figure 1. Time-vertical section of near-inertial velocity $\left(\mathrm{m} \mathrm{s}^{-1}\right)$ : (top) downward- and (bottom) upward-propagating components. (left) Vertical profiles of time-averaged Brunt-Väisälä frequency $\left(\mathrm{h}^{-1}\right)$ and WKB stretched depth $(\mathrm{sm})$. Near-inertial waves trapped inside a baroclinic eddy principally traveled downward, and partially reflected to the surface near the intermediate density gap (dashed horizontal).

\section{References}

Kawaguchi, Y., S. Nishino, J. Inoue, K. Maeno, H. Takeda, and K. Oshima, Enhanced diapycnal mixing due to near-inertial internal waves propagating through an Anticyclonic eddy in the ice-free Chukchi Plateau, J. Phys. Oceanogr., 46, 2457-25481, 2016. 